\title{
A NOVEL APPROACH TO THERMAL AND MECHANICAL STRESSES IN A FGM CYLINDER WITH EXPONENTIALLY-VARYING PROPERTIES
}

\author{
Kerimcan Celebi \\ Adana Science and Technology University, Department of Mechanical Engineering, Adana, Turkey \\ DURMUS YARIMPABUC \\ Osmaniye Korkut Ata University, Department of Mathematics, Osmaniye, Turkey \\ e-mail: durmusyarimpabuc@osmaniye.edu.tr \\ IBRAHIM KELES \\ Department of Mechanical Engineering, Ondokuz Mayis University, Samsun, Turkey
}

\begin{abstract}
A novel approach is employed to a general solution for one-dimensional steady-state thermal and mechanical stresses in a hollow thick cylinder made of a functionally graded material (FGM). The temperature distribution is assumed to be a function of radius, with general thermal and mechanical boundary conditions on the inside and outside surfaces of the cylinder. The material properties, except Poisson's ratio, are assumed to be exponentially-varying through the thickness. Forcing functions applied to the inner boundary are internal pressures which may be in form of steps. These conditions result in governing differential equations with variable coefficients. Analytical solutions to such equations cannot be obtained except for certain simple grading functions and pressures. Numerical approaches must be adopted to solve the problem in hand. The novelty of the present study lies in the fact that the Complementary Functions Method (CFM) is employed in the analysis. The Complementary Functions method (CFM) will be infused into the analysis to convert the problem into an initial-value problem which can be solved accurately. Benchmark solutions available in the literature are used to validate the results and to observe the convergence of the numerical solutions. The solution procedure is well-structured, simple and efficient and it can be readily applied to cylinders. It is also well suited for problems in which mechanical properties are graded.
\end{abstract}

Keywords: thermal stresses, functionally-graded materials, thick cylinder, Complementary Functions Method

\section{Introduction}

Pressure vessel structural members such as cylinders, disks and spheres find broad application fields in the industry, and their vibration analyses are deemed necessary for safe design and operation. Hollow cylinders and thick-walled cylindrical shells are common components in structural applications and device systems involving aerospace and submarine structures, civil engineering structures, machines, pipes, sensors and actuators, etc. These structures are often exposed to temperature environment and thermal stresses are then induced. In many cases, thermal stresses will significantly depress strength and also affect functionality of structures. Thus, the exact analysis of thermal stresses is really important (Ying and Wang, 2010). There have been many studies, such as Timoshenko and Woinowsky-Krieger (1959), Boley and Weiner (1960), Das and Navaratna (1962), Das and Rath (1972), Stavsky (1963) and Thangaratnam et al. (1988), which focused on thermal stresses in isotropic homogeneous rectangular plates. Yee and Moon (2002) have been obtained a closed-form analytical solution for the plane thermal stress analysis of a homogeneously orthotropic hollow cylinder subjected to an arbitrary, transient, asymmetric 
temperature distribution. They used a stress function approach for obtaining hoop, radial, and shear stresses in a hollow cylinder. Shao (2005) presented, by using a multi-layered approach based on the theory of laminated composites, the solutions of temperature, displacements, and thermal-mechanical stresses in a functionally graded circular hollow cylinder. Shao et al. (2008) used complex Fourier series and Laplace transform techniques to investigate transient heat conduction and thermo-mechanical stresses in an FGM hollow cylinder. Jabbari et al. (2002, 2003, 2009) derived the exact solution for one-dimensional and two dimensional steady-state thermoelastic problems of functionally graded hollow cylinders where material properties varied with the power product form of the radial coordinate variable. Recently Ruhi et al. (2005) studied thermoelastic analysis of thick walled finite length cylinders of functionally graded materials and achieved results for stress, strain and displacement components through the thickness and along the length. The results were presented for uniform internal pressure and thermal loading. Ootao and Tanigawa (2006) analyzed exactly a one-dimensional transient thermoelastic problem of a functionally graded hollow cylinder whose thermal and thermoelastic constants were assumed to vary with the power product form of the radial coordinate variable. The resulting governing differential equation then possessed variable coefficients. General closed-form solutions to such equations are not available. Noda et al. (2012) studied the transient thermoelastic analysis for an FGM solid circular disk whose material properties were expressed by a piecewise power law. As it was done in the works cited above, in such situations the solution methods included integral transformations, development of finite element models, and, in some special cases, series solutions were attempted. Assuming that the member was composed of many homogeneous layers of different properties emulating the FGM behavior, there was another way of tackling the problem on hand. All of these approaches required heavy mathematical manipulations and, in the case of having to discretize the domain into many elements, a high amount of computational time.

In the present paper, the governing differential equation is non-homogeneous with variable coefficients which include material properties. A novel approach is attempted to obtain displacements, strains and stresses in a simple and efficient manner. The complementary functions method (CFM), theoretically explained in the literature by Aktaş (1972), Agarwal (1982) and Roberts and Shipman (1979) is infused into analysis to convert the problem to an initial-value problem which can be then easily solved by, for example, the fifth-order Runge-Kutta method (RK5) with great accuracy (Chapra and Canale, 1998). Shell theories or dividing the material into homogeneous subelements of different properties emulating the graded behavior contains the customary approach of modeling FGM structural elements. Finite element analysis, series expansion methods and direct methods are primary solution methods used in the literature. The present paper uses a novel and efficient method which employes CFM. A thick hollow cylinder of FGM under one-dimensional steady-state temperature distribution with general types of thermal and mechanical boundary conditions is analysed. Two material models are used: (a) with a simple power law with constant Poisson's ratio (Jabbari et al., 2002) for which analytical benchmark solutions are available, (b) with exponentially-varying properties. It should be emphasized once again that the solution procedure is not confined to any particular choice of the material model; it is equally suitable for arbitrary functions defining the gradient variation of material properties.

\section{Solutions by the Complementary Functions Method}

The CFM transforms two-point boundary-value problems to a system of initial-value problems. It reduces to a particularly simple solution scheme when applied to a given class of problems, e.g. for an annular disk of inner radius $r_{i}$ and outer radius $r_{o}$. As it is shown in the proceeding Sections, under axisymmetric conditions, the governing differential equation of the dependent variable $u(r)$ in its most general form is 


$$
u^{\prime \prime}+P(r) u^{\prime}+Q(r) u=R(r)
$$

subject to boundary conditions on the inner $\left(r=r_{i}\right)$ and outer $\left(r=r_{o}\right)$ surfaces. Here $(\cdot)^{\prime}$ denotes the derivative with respect to $r$. A general closed-form solution of the above equation cannot be obtained. The complete solution to Eq. (2.1) is

$$
u=b_{j} u_{j}+u_{p} \quad j=1,2
$$

where $u_{j}$ and $u_{p}$ are, respectively, homogenous and particular solutions. The coefficients $b_{j}$ are determined via the boundary conditions. CFM begins by assuming $u_{i}=Y_{1}^{(i)}$ and $u_{i}^{\prime}=Y_{2}^{(i)}$, which means

$$
\left(Y_{1}^{(i)}\right)^{\prime}=Y_{2}^{(i)}
$$

Here, the index $i=1,2$ refers to homogeneous solutions and $i=p$ means the particular solution. To determine the homogeneous solutions, the right-hand side of Eq. (2.1) is set equal to zero, and the following is obtained

$$
\left(Y_{2}^{(i)}\right)^{\prime}=-P(r) Y_{2}^{(i)}-Q(r) Y_{1}^{(i)}
$$

The system of Eqs. (2.3) and (2.4) can be solved numerically for each homogeneous solution. The Kronecker delta initial conditions given below are used to assure linear independence of the solutions (Roberts and Shipman, 1979)

$$
Y_{j}^{(i)}=\delta_{j i} \quad j, i=1,2
$$

To obtain the particular solution, Eq. (2.4) is modified as

$$
\left(Y_{2}^{(p)}\right)^{\prime}=-P(r) Y_{2}^{(p)}-Q(r) Y_{1}^{(p)}+R(r)
$$

A particular solution needs only to satisfy the differential equation and homogeneous initial conditions

$$
Y_{j}^{(p)}=0 \quad j=1,2
$$

be imposed. Equations (2.3), (2.6), (2.7) constitute a system of equations for the particular solution along with the initial conditions. The fifth-order Runge-Kutta method (RK5) is used for all cases considered. Note that by this procedure not only the solution $u(r)$ itself but also its first derivative are readily calculated. Applying the boundary conditions prescribed for the particular problem in hand results in the following system of algebraic equations for the coefficients $b_{1}$ and $b_{2}$

$$
\left[\begin{array}{ll}
A_{11} & A_{12} \\
A_{21} & A_{22}
\end{array}\right]\left[\begin{array}{l}
b_{1} \\
b_{2}
\end{array}\right]=\left[\begin{array}{l}
R H S 1 \\
R H S 2
\end{array}\right]
$$

Here, $A_{i j}$ includes the values of the homogeneous solutions at the boundary points. $R H S 1$ and $R H S 2$ contain values of the particular solutions. If the cylinder is subjected to internal and external pressures, they will also be included in the right hand-side terms. On the other hand, implementing CFM in the heat conduction problem yields $R H S 1$ and $R H S 2$ as prescribed temperatures along the boundaries. These points will be illustrated in the following Sections. 


\section{Heat conduction in the radial direction}

The heat conduction equation in the steady-state condition for a one-dimensional problem in polar coordinates and thermal boundary conditions for a FGM hollow cylinder are given, respectively, as

$$
\begin{aligned}
& \frac{1}{r}\left(r k(r) T^{\prime}(r)\right)^{\prime}=0 \quad r_{i} \leqslant r \leqslant r_{o} \\
& C_{11} T\left(r_{i}\right)+C_{12} T^{\prime}\left(r_{i}\right)=f_{1} \\
& C_{11} T\left(r_{o}\right)+C_{12} T^{\prime}\left(r_{o}\right)=f_{2}
\end{aligned}
$$

where $k=k(r)$ is the thermal conduction coefficient, $r_{i}$ and $r_{o}$ are the inner and outer radii of the hollow cylinder. $C_{i j}$ are the constant thermal parameters related to the conduction and convection coefficients. The constants $f_{1}$ and $f_{2}$ are known constants on the inside and outside radii.

It is assumed that the nonhomogeneous thermal conduction coefficient $k(r)$ is an exponential function of $r$ as

$$
k(r)=k_{o} \mathrm{e}^{\beta r}
$$

where $k_{o}$ is a material constant and $\beta$ is the inhomogeneity parameter. Using Eq. (3.2), the heat conduction equation becomes

$$
\frac{1}{r}\left(r \mathrm{e}^{\beta r} T^{\prime}(r)\right)^{\prime}=0
$$

Steady-state axisymmetric heat conduction without heat generation is considered. The heat balance equation in the radial direction for a nonuniform disk yields

$$
T^{\prime \prime}+B(r) T^{\prime}=0
$$

where $B(r)=(1 / r)+\beta$ and it is varying as a function of the radial coordinate $r$. The boundary conductions are temperatures prescribed on the inner and outer surfaces as

$$
T\left(r_{i}\right)=T_{i} \quad \text { and } \quad T\left(r_{o}\right)=T_{o}
$$

The complete solution is the homogeneous solution

$$
T=b_{j} T_{j} \quad j=1,2
$$

with

$$
T^{\prime}=b_{j} T_{j}^{\prime} \quad j=1,2
$$

Following the steps outlined in Section 2, the temperature distribution is obtained at the collocation points. The constants $b_{j}$ can now be found by imposing the boundary conditions. This process results in a system given by Eq. (2.8) where

$$
\begin{array}{ll}
A_{11}=T_{1}\left(r_{i}\right) & A_{12}=T_{2}\left(r_{i}\right) \\
A_{21}=T_{1}\left(r_{o}\right) & A_{22}=T_{2}\left(r_{o}\right) \\
R H S 1=T_{i} & \text { RHS } 2=T_{o}
\end{array}
$$




\section{Governing equation}

Consider a thick walled cylinder of the inside radius $r_{i}$ and the outside radius $r_{o}$ made of FGM. The material is graded through the $r$-direction. Let $u$ be the displacement component in the radial direction. Then the strain-displacement relations are

$$
\varepsilon_{r r}=\frac{d u}{d r} \quad \varepsilon_{\theta \theta}=\frac{u}{r}
$$

The stress-strain relations are

$$
\begin{aligned}
& \sigma_{r r}=(\lambda+2 \mu) \varepsilon_{r r}+\lambda \varepsilon_{\theta \theta}-(3 \lambda+2 \mu) \alpha T(r) \\
& \sigma_{\theta \theta}=(\lambda+2 \mu) \varepsilon_{\theta \theta}+\lambda \varepsilon_{r r}-(3 \lambda+2 \mu) \alpha T(r)
\end{aligned}
$$

where $\sigma_{i j}$ and $\varepsilon_{i j}(i, j=r, \theta)$ are stress and strain tensors, $T(r)$ is temperature distribution determined from the heat conduction equation, $\alpha$ is the coefficient of thermal expansion, and $\lambda$ and $\mu$ are the Lame coefficients related to the modulus of elasticity $E$ and Poisson's ratio $\nu$ as

$$
\lambda=\frac{\nu E(r)}{(1+\nu)(1-2 \nu)} \quad \mu=\frac{E(r)}{2(1+\nu)}
$$

The equilibrium equation in the radial direction, disregarding the body force and inertia terms, is

$$
\frac{\partial \sigma_{r r}}{\partial r}+\frac{\sigma_{r r}-\sigma_{\theta \theta}}{r}=0
$$

To obtain the equilibrium equation in terms of the displacement component for the FGM cylinder, the functional relationship of the material properties must be known. To ascertain the effect of the inhomogeneity, the properties are considered to vary exponentially across the thickness

$$
E(r)=E_{o} \mathrm{e}^{\beta r} \quad \alpha=\alpha_{o} \mathrm{e}^{\beta r}
$$

where $E_{o}$ and $\alpha_{o}$ are the material constants and $\beta$ is the inhomogeneity parameter. Poisson's ratio varies very little through the thickness in FGM materials. Furthermore, its effects on thermal and mechanical stresses are insignificant. For simplicity, Poisson's ratio is assumed to be constant (Akbari Alashti et al., 2013; Jabbari et al., 2015).

Using relations (4.1)-(4.5), the Navier equation in term of the displacement is

$$
u^{\prime \prime}+P(r) u^{\prime}+Q(r) u=R(r)
$$

where

$$
\begin{aligned}
& P(r)=(\beta r+1) \frac{1}{r} \quad Q(r)=\left(\frac{\nu \beta r}{1-\nu}-1\right) \frac{1}{r^{2}} \\
& R(r)=\frac{\mathrm{e}^{\beta r} \alpha_{o}(1+\nu)}{(1-\nu)}\left(2 \beta T+T^{\prime}\right)
\end{aligned}
$$

Following the steps outlined in Section 2, the complete displacement is obtained at the collocation points as

$$
u=b_{1} u_{1}+b_{2} u_{2}+u_{p}
$$

with

$$
u^{\prime}=b_{1} u_{1}^{\prime}+b_{2} u_{2}^{\prime}+u_{p}^{\prime}
$$

The coefficients $b_{1}, b_{2}$ will be determined using the stress free conditions on inner $\left(\sigma_{r r}\left(r_{i}\right)=-P_{i}\right)$ and outer $\left(\sigma_{r r}\left(r_{o}\right)=-P_{o}\right)$ boundaries. This step is particularly simple since during the solution process the first derivative of the radial displacement has already been calculated. 


\section{Results and discussions}

As an example, consider a thick hollow cylinder of the inner radius a $r_{i}=1 \mathrm{~m}$ and the outer radius $r_{o}=1.2 \mathrm{~m}$. Poisson's ratio is taken to be 0.3 , and the modulus of elasticity and the thermal coefficient of expansion at the inner radius are $E_{o}=200 \mathrm{GPa}$ and $\alpha_{o}=1.2 \cdot 10^{-6} /{ }^{\circ} \mathrm{C}$, respectively. The properties are considered to vary exponentially across the thickness. The boundary conditions for temperature are taken as $T\left(r_{i}\right)=10^{\circ} \mathrm{C}$ and $T\left(r_{o}\right)=0{ }^{\circ} \mathrm{C}$. The hollow cylinder has pressure on its inner surface, so the boundary conditions for stresses are assumed as $\sigma_{r r}\left(r_{i}\right)=-50 \mathrm{MPa}$ and $\sigma_{r r}\left(r_{o}\right)=0 \mathrm{MPa}$.

The numerical solution in the present study is checked with the results obtained by Jabbari et al. (2002) for the validation purpose. Comparison is illustrated in Tables 1-3. It can be observed that the results are in good agreement with the same results by Jabbari et al. (2002). The numerical results have been obtained to six-digit accuracy by picking only 11 collocation points.

Table 1. Comparison of CFM with Jabbari et al. (2002) for a homogenous cylinder $(m=0$ and $\beta=0)$

\begin{tabular}{|c|c|c|c|c|c|c|c|c|}
\hline \multirow{2}{*}{$r_{i}$} & \multicolumn{2}{|c|}{$T / T\left(r_{i}\right)$} & \multicolumn{2}{c|}{$u / r_{i}$} & \multicolumn{2}{c|}{$\sigma_{r r} / P_{i}$} & \multicolumn{2}{c|}{$\sigma_{\theta \theta} / P_{i}$} \\
\cline { 2 - 9 } & CFM & Jabbari & CFM & Jabbari & CFM & Jabbari & CFM & Jabbari \\
\hline \hline 1 & 1 & 1 & 0.00136642 & 0.00136642 & -1 & -1 & 5.50909 & 5.50909 \\
\hline 1.04 & 0.784882 & 0.784882 & 0.00133799 & 0.00133799 & -0.754183 & -0.754183 & 5.27802 & 5.27802 \\
\hline 1.08 & 0.577883 & 0.577883 & 0.00131239 & 0.00131239 & -0.534644 & -0.534644 & 5.07268 & 5.07268 \\
\hline 1.12 & 0.378413 & 0.378413 & 0.00128932 & 0.00128932 & -0.337716 & -0.337716 & 4.88943 & 4.88943 \\
\hline 1.16 & 0.185944 & 0.185944 & 0.0012685 & 0.0012685 & -0.160351 & -0.160351 & 4.72526 & 4.72526 \\
\hline 1.2 & 0 & 0 & 0.0012497 & 0.0012497 & 0 & 0 & 4.57766 & 4.57766 \\
\hline
\end{tabular}

Table 2. Comparison of CFM with Jabbari et al. (2002) for FGM cylinders with constant Poisson's ratio and the elastic modulus obeying a simple power law $(m=-2$ and $\beta=-2)$

\begin{tabular}{|c|c|c|c|c|c|c|c|c|}
\hline \multirow{2}{*}{$r_{i}$} & \multicolumn{2}{|c|}{$T / T\left(r_{i}\right)$} & \multicolumn{2}{c|}{$u / r_{i}$} & \multicolumn{2}{c|}{$\sigma_{r r} / P_{i}$} & \multicolumn{2}{c|}{$\sigma_{\theta \theta} / P_{i}$} \\
\cline { 2 - 9 } & CFM & Jabbari & CFM & Jabbari & CFM & Jabbari & CFM & Jabbari \\
\hline \hline 1 & 1 & 1 & 0.00161944 & 0.00161944 & -1 & -1 & 6.62127 & 6.62127 \\
\hline 1.04 & 0.814545 & 0.814545 & 0.00158667 & 0.00158667 & -0.722291 & -0.722291 & 5.84288 & 5.84288 \\
\hline 1.08 & 0.621818 & 0.621818 & 0.00155679 & 0.00155679 & -0.491571 & -0.491571 & 5.19022 & 5.19022 \\
\hline 1.12 & 0.421818 & 0.421818 & 0.00152961 & 0.00152961 & -0.29876 & -0.29876 & 4.63928 & 4.63928 \\
\hline 1.16 & 0.214545 & 0.214545 & 0.00150494 & 0.00150494 & -0.136767 & -0.136767 & 4.1713 & 4.1713 \\
\hline 1.2 & 0 & 0 & 0.00148263 & 0.00148263 & 0 & 0 & 3.77145 & 3.77145 \\
\hline
\end{tabular}

Table 3. Comparison of CFM with Jabbari et al. (2002) for FGM cylinders with constant Poisson's ratio and the elastic modulus obeying a simple power law $(m=2$ and $\beta=2)$

\begin{tabular}{|c|c|c|c|c|c|c|c|c|}
\hline \multirow{2}{*}{$\frac{r}{r_{i}}$} & \multicolumn{2}{|c|}{$T / T\left(r_{i}\right)$} & \multicolumn{2}{c|}{$u / r_{i}$} & \multicolumn{2}{c|}{$\sigma_{r r} / P_{i}$} & \multicolumn{2}{c|}{$\sigma_{\theta \theta} / P_{i}$} \\
\cline { 2 - 9 } & CFM & Jabbari & CFM & Jabbari & CFM & Jabbari & CFM & Jabbari \\
\hline \hline 1 & 1 & 1 & 0.00114082 & 0.00114082 & -1 & -1 & 4.51743 & 4.51743 \\
\hline 1.04 & 0.753093 & 0.753093 & 0.00111628 & 0.00111628 & -0.784165 & -0.784165 & 4.70652 & 4.70652 \\
\hline 1.08 & 0.533109 & 0.533109 & 0.00109454 & 0.00109454 & -0.577254 & -0.577254 & 4.89893 & 4.89893 \\
\hline 1.12 & 0.336271 & 0.336271 & 0.00107516 & 0.00107516 & -0.378189 & -0.378189 & 5.09474 & 5.09474 \\
\hline 1.16 & 0.159442 & 0.159442 & 0.00105779 & 0.00105779 & -0.186042 & -0.186042 & 5.29405 & 5.29405 \\
\hline 1.2 & 0 & 0 & 0.00104213 & 0.00104213 & 0 & 0 & 5.49697 & 5.49697 \\
\hline
\end{tabular}


Figure 1a shows variations of temperature along the radial direction for different values of the inhomogeneity parameter $(\beta)$. The figure shows that as the inhomogeneity parameter $\beta$ increases, the temperature decreases. Figure $1 \mathrm{~b}$ shows the plot of the radial displacement along the radius. The magnitude of the radial displacement is decreased as the inhomogeneity parameter $\beta$ is increased. The radial and circumferential stresses are plotted along the radial direction and are shown in Figs. 1c and 1d. The magnitude of the radial stress is increased as $\beta$ is increased. It is seen that for $\beta<1$ the hoop stress decreases along the radial direction. For $\beta>1$, the hoop stress increases as the radius increases, since the modulus of elasticity is an increasing function of the radius, see Eq. (2.6). Physically, this means that the outer layers of the cylinder are biased to maintain the stress due to their higher stiffness. There is a limiting value for $\beta$, where the hoop stress remains almost constant along the radius. The curve associated with $\beta=1$ shows that the variation of hoop stress along the radial direction is minor, and is almost uniform across the radius. To investigate the pattern of stress distribution along the cylinder radius, the effective stress $\sigma^{*}=\sqrt{2}\left|\sigma_{r}-\sigma_{\theta}\right|$ is plotted along the radial direction for different values of $r_{o} / r_{i}$ and the inhomogeneity parameter $\beta$. Figure 2 is plotted for $r_{o} / r_{i}=1.2$. It is interesting to note from Fig. 2 that for $\beta=1$ the effective stress is almost uniform along the radius of the cylinder.

(a)

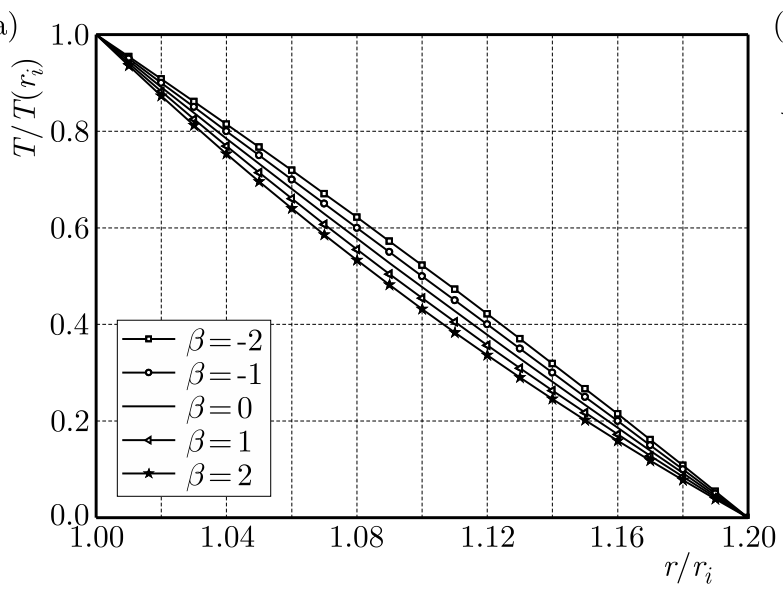

(b)

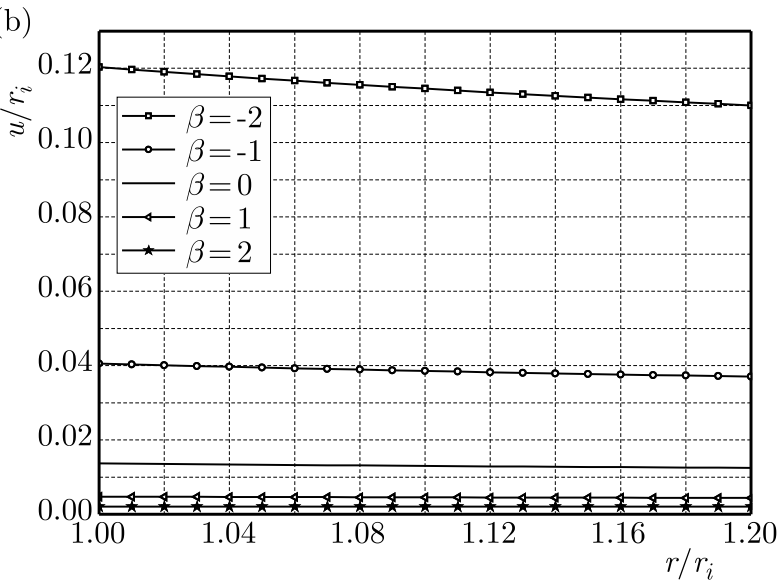

(c)

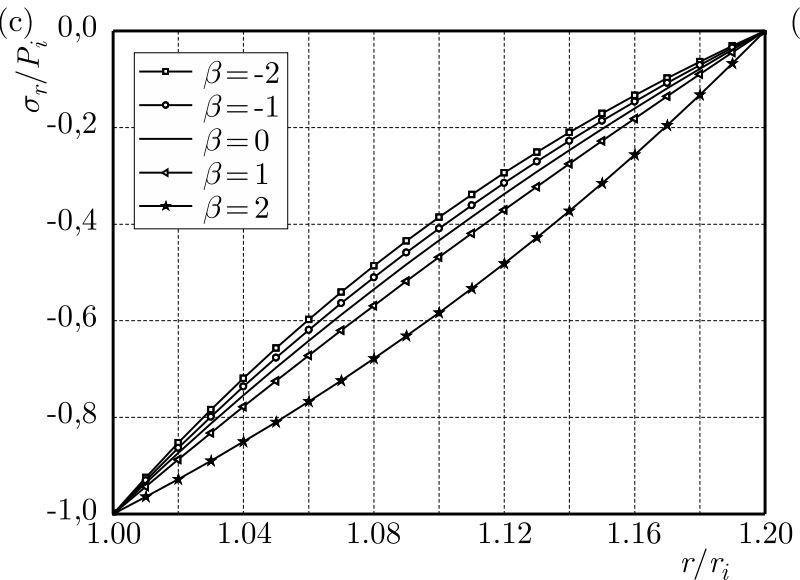

(d)

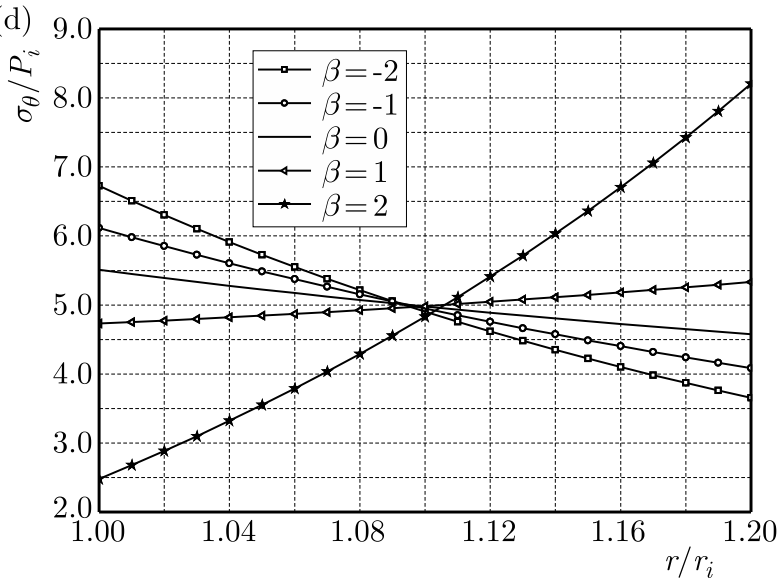

Fig. 1. Radial distribution of: (a) temperature, (b) radial displacement, (c) radial stress and (d) hoop stress for cylinder

It should be pointed out once again that the purpose of the present work is the introduction of CFM to the solution procedure of the class of problems in hand. Converting the two-point boundary value problem to a system of an initial-value problem gives a way to the implementation of well-established numerical schemes. The Runge-Kutta method of fifth-order (RK5) is used to 


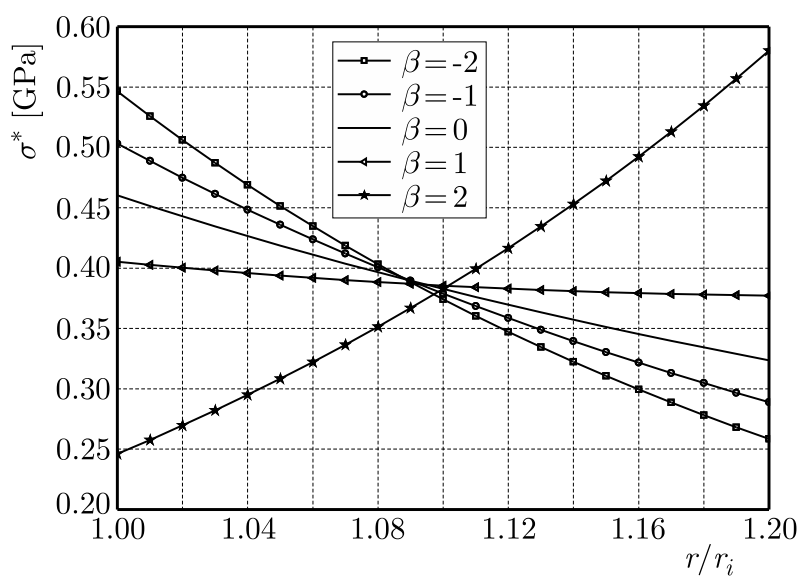

Fig. 2. Effective stress distribution for $r_{o} / r_{i}=1.2$

solve the system of equations. The procedure is simple and efficiently implemented. The numerical results have been obtained exact up to six-digit accuracy by picking only 11 collocation points in RK5.

\section{Conclusion}

This paper presents a numerical solution for calculation of axisymmetric thermal and mechanical stresses in a thick hollow cylinder made of FGM. The material properties through the graded direction are assumed to be nonlinear with a power law distribution and exponentially-varying properties. The mechanical and thermal stresses are obtained through the CFM of the solution of the Navier equation. The comparisons of temperature distributions and stress distributions are presented in form of tables. The numerical results for all cases are shown to exactly match those reported by Jabbari et al. (2002). Finally, we can conclude that:

- With the unified approach presented in the present study, one would not have to compromise on the functional continuity of the material properties. Analysis of any material model in form of an arbitrary function subject to internal pressure has been analyzed efficiently and accurately by employing CFM.

- The unified method used is accurate and more efficient than the conventional methods.

- The method employed in this study allows one to find solutions of continuous functions.

- The CFM of solving the differential equation provides a complete solution, yielding both thermal stresses and temperature distributions.

\section{References}

1. Agarwal R.P., 1982, On the method of complementary functions for nonlinear boundary-value problems, Journal of Optimization Theory and Applications, 36, 1, 139-144

2. Akbari Alashti R., Khorsand M., Tarahhomi M.H., 2013, Three-dimensional asymmetric thermo-elastic analysis of a functionally graded rotating cylindrical shell, Journal of Theoritical and Applied Mechanics, 51, 1, 143-158

3. Aktaş Z., 1972, Numerical Solutions of Two-Point Boundary Value Problems, Ankara Turkey: METU, Dept. of Computer Engineering

4. Boley B.A., Weiner J.H., 1960, Theory of Thermal Stresses, Wiley Hoboken, New Jork, 35-45 
5. Chapra S.C., Canale R.P., 1998, Numerical Methods for Engineers, 2nd ed., McGraw-Hill, New York, 760-766

6. Das Y.C., Navaratna D.R., 1962, Thermal bending of rectangular plates, Journal of the Aerospace Sciences, 29, 11, 1397-1399

7. Das Y.C., RAth B.K., 1972, Thermal bending of moderately thick rectangular plates, AIAA Journal, 10, 10, 1349-1351

8. Jabbari M., Bahtui A., Eslami M.R., 2009, Axisymmetric mechanical and thermal stresses in thick short length FGM cylinders, International Journal of Pressure Vessels and Piping, 86, 5, 296-306

9. Jabbari M., NeJad M.Z., Ghannad M., 2015, Thermo-elastic analysis of axially functionally graded rotating thick cylindrical pressure vessels with variable thickness under mechanical loading, International Journal of of Engineering Science, 96, 1-18

10. Jabbari M., Sohrabpour S., Eslami M.R., 2002, Mechanical and thermal stresses in a functionally graded hollow cylinder due to radially symmetric loads, International Journal of Pressure Vessels and Piping, 79, 7, 493-497

11. Jabbari M., Sohrabpour S., Eslami M.R., 2003, General solution for mechanical and thermal stresses in a functionally graded hollow cylinder due to nonaxisymmetric steady-state loads, ASME Journal of Applied Mechanics, 70, 1, 111-118

12. Noda N., Ootao Y., Tanigawa Y., 2012, Transient thermoelastic analysis for functionally graded circular disk with piecewise power law, Journal of Theoretical and Applied Mechanics, 50, $3,831-839$

13. Ootao Y., Tanigawa Y., 2006, Transient thermoelastic analysis for functionally graded hollow cylinder, Journal of Thermal Stresses, 29, 11, 1031-1046

14. Roberts S.M., Shipman J.S., 1979, Fundamental matrix and two-point boundary-value problems, Journal of Optimization Theory and Applications, 28, 1, 77-78

15. Ruhi M., Angoshtari A., Naghdabadi R., 2005, Thermoelastic analysis of thick-walled finite-length cylinders of functionally graded materials, Journal of Thermal Stresses, 28, 4, 391-408

16. Shao Z.S., 2005, Mechanical and thermal stresses of a functionally graded circular hollow cylinder with finite length, International Journal of Pressure Vessels and Piping, 82, 3, 155-163

17. Shao Z.S., Ang K.K., Reddy J.N., Wang T.J., 2008, Nonaxisymmetric thermomechanical analysis of functionally graded hollow cylinders, Journal of Thermal Stresses, 31, 6, 515-536

18. Stavsky Y., 1963, Thermoelasticity of heterogeneous aeolotropic plates, Journal of the Engineering Mechanics Division, 89, 2, 89-105

19. Thangaratnam R.K., Palaninathan, Ramachandran J., 1988, Thermal stress analysis of laminated composite plates and shells, Computers and Structures, 30, 6, 1403-1411

20. Timoshenko S., Woinowsky-Krieger S., 1959, Theory of Plates and Shells, 2nd ed., McGraw-Hill, New York, 73-132

21. Yee K.C., Moon T.J., 2002, Plane thermal stress analysis of an orthotropic cylinder subjected to an arbitrary, transient, axisymmetric tempherature distribution, ASME Journal of Applied Mechanics, 69, 5, 632-640

22. Ying J., WAng H.M., 2010, Axisymmetric thermoelastic analysis in a finite hollow cylinder due to nonuniform thermal shock, International Journal of Pressure Vessels and Piping, 87, 12, 714-720 